\title{
Nanoplasmonics in the TEM
}

\author{
Michel Bosman
}

Institute of Materials Research and Engineering, A*STAR (Agency for Science, Technology and Research), 3 Research Link, Singapore 117602

This paper reviews recent developments in scanning TEM (STEM)-based characterization of localized surface plasmons. It will be shown that localized surface plasmon resonances can be mapped with STEM cathodoluminescence as well as with monochromated electron energy-loss spectroscopy (EELS) [1]. The spectral and spatial resolution of the techniques will be discussed, and examples will be given that demonstrate the unique capability of STEM-based plasmon analysis in comparison with other experimental techniques.

Plasmon resonances on metal surfaces can be excited by light, transforming it from a free-space wave to a surface-bound oscillation. The modulation of light through surface plasmon resonances promises a range of novel technological applications, including nonlinear optics and fast circuitry.

Fabrication and synthesis techniques are showing remarkable progress in obtaining nanometer precision in size and morphology of metal structures and particles, but characterization techniques typically lack this accuracy. An exception is STEM-based characterization, which has a long history in plasmon characterization [2-5], but has only recently become more widely used [6-10] thanks to instrumental developments $[11,12]$.

The first advantage of using STEM for surface plasmon characterization is the high spatial accuracy that the technique provides. We will show that in addition, EELS analysis will also give a more complete picture of the nature of the plasmon resonances. After all, far field optical characterization will mostly measure bright plasmon modes. With STEM on the other hand, highly localized fields surround the STEM probe, making this a clear near-field technique. As a result, bright as well as dark plasmon resonant modes can be excited, allowing a full-modal characterization, as shown for example in Figure 1 below [12].

The second advantage of this technique, is the possibility of measuring both atomic-resolution (S)TEM images and local spectra at the same location. A systematic series of measurements can now be obtained by doing monochromated EELS at several sample locations where the particle morphology and interparticle distances are measured. Even when the sample fabrication or particle synthesis does not yield a $100 \%$ success rate, measurements at selected locations will still give a full picture of the plasmon response, something that would not be possible with far-field techniques that measure many structures simultaneously over large areas. By combining imaging and local plasmon spectroscopy, we will demonstrate the occurrence of plasmon-enhanced electron tunneling [14, 15], and we will present in detail a method to control this novel phenomenon with the help of molecular monolayers [15]. The latter results are important not just as proof for the occurrence of plasmon-enhanced electron tunneling, but also as a demonstration how monochromated STEM-EELS can be used to measure the THz electronic properties of molecules. 
References:

[1] A Losquin et al., Nano Lett. DOI: 10.1021/n15043775 (2015).

[2] C Powell and JB Swan, Phys. Rev. 115 (1959),p. 869.

[3] PE Batson, Phys. Rev. Lett. 49 (1982), p. 936.

[4] ZL Wang, JM Cowley, Ultramicrosopy 21 (1987), p. 347

[5] F Ouyang, PE Batson and M Isaacson, Phys. Rev. B 46 (1992), p. 15421.

[6] J Nelayah et al., Nature Phys. 3 (2007), p. 348.

[7] M Bosman et al., Nanotechnology 18 (2007), p. 165505.

[8] B Schaffer et al., Phys. Rev. B (2009), p. 041401

[9] H Duan et al. Nano Lett. 12 (2012), p. 683.

[10] D Rossouw and GA Botton, Phys. Rev. Lett. 110 (2013), p. 066801.

[11] PC Tiemeijer, Ultramicroscopy 78 (1999), p. 53.

[12] OL Krivanek et al. Nature 514 (2014), p. 209.

[13] A Teulle et al. Nature Materials 14 87-94 (2015).

[14] DC Marinica et al. Nano Lett. 12 (2012), p. 1333.

[15] JA Scholl et al. Nano Lett. 13 (2013), p. 564.

[16] SF Tan et al. Science 343 (2014), p. 1496.
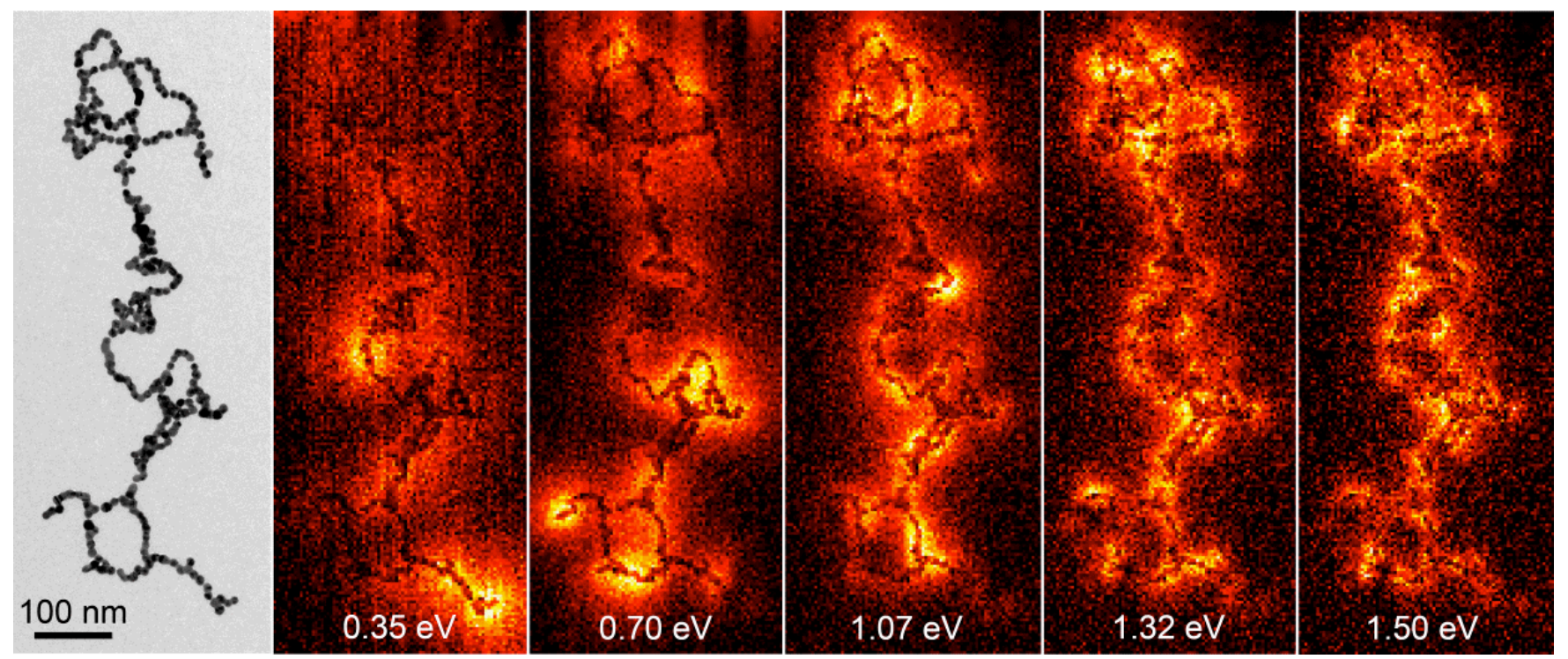

Figure 1. TEM image (left) showing a fused network of gold nanoparticles, each about $12 \mathrm{~nm}$ in diameter. The monochromated EELS maps (panels 2-6) show various infrared plasmon modes that are resonant in this network. The energy values at the bottom of each map indicate the resonant energy at which the plasmon mode is mapped for each panel. It can be seen that plasmon modes can resonate over relatively large distances of several hundreds of nanometers; see also [13]. 